\title{
The intersystem crossing of a cyclic (alkyl)(amino) carbene gold (i) complex
}

S. Thompson, J. Eng, and T. J. Penfold

Citation: The Journal of Chemical Physics 149, 014304 (2018); doi: 10.1063/1.5032185

View online: https://doi.org/10.1063/1.5032185

View Table of Contents: http://aip.scitation.org/toc/jcp/149/1

Published by the American Institute of Physics

\section{Articles you may be interested in}

Announcement: Top reviewers for The Journal of Chemical Physics 2017

The Journal of Chemical Physics 149, 010201 (2018); 10.1063/1.5043197

Mean-field Matsubara dynamics: Analysis of path-integral curvature effects in rovibrational spectra

The Journal of Chemical Physics 149, 014102 (2018); 10.1063/1.5038616

Large-scale relativistic complete active space self-consistent field with robust convergence

The Journal of Chemical Physics 149, 014106 (2018); 10.1063/1.5036594

Complete active space configuration interaction from state-averaged configuration interaction singles natural orbitals: Analytic first derivatives and derivative coupling vectors

The Journal of Chemical Physics 147, 094104 (2017); 10.1063/1.5000476

Analytic gradient and derivative couplings for the spin-flip extended configuration interaction singles method:

Theory, implementation, and application to proton transfer

The Journal of Chemical Physics 148, 244108 (2018); 10.1063/1.5037081

Density functional theory calculations for magnetic properties of $\mathrm{C}_{3} \mathrm{~W}$ systems

The Journal of Chemical Physics 149, 014303 (2018); 10.1063/1.5029398

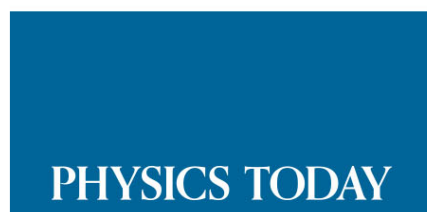




\title{
The intersystem crossing of a cyclic (alkyl)(amino) carbene gold (I) complex
}

\author{
S. Thompson, J. Eng, ${ }^{\text {a) }}$ and T. J. Penfold ${ }^{\text {b) }}$ \\ Chemistry, School of Natural and Environmental Sciences, Newcastle University, \\ Newcastle upon Tyne NE1 7RU, United Kingdom
}

(Received 3 April 2018; accepted 11 June 2018; published online 5 July 2018)

\begin{abstract}
The intersystem crossing (ISC) mechanism of a cyclic (alkyl)(amino) carbene gold (I) complex (CMA1) is studied using quantum dynamics. A model spin-vibronic Hamiltonian is developed, which includes 10 excited states and two important nuclear degrees of freedom. The quantum dynamics reveals that ISC from $S_{1} \rightarrow T_{1}$ occurs on the tens of picosecond time scale, consistent with recent experiments. It is driven by motion along the torsional degree of freedom of the carbazole $(\mathrm{Cz})$ ligand, which causes orthogonality between the donor and acceptor groups closing the gap between the initial $\left(\mathrm{S}_{1}\right)$ and final $\left(\mathrm{T}_{1}\right)$ states. The role of higher triplet states through spin-vibronic interactions is also discussed. Although previous calculations, evaluated in the Condon approximation, yield large ISC rates, our present dynamical treatment, taking into account the large amplitude torsional motion, increases the calculated rate by an order of magnitude improving the agreement with experiments. The model spin-vibronic Hamiltonian developed can also be used to understand the properties of related linear metal carbene compounds, facilitating molecular design. (C) 2018 Author(s). All article content, except where otherwise noted, is licensed under a Creative Commons Attribution (CC BY) license (http://creativecommons.org/licenses/by/4.0/). https://doi.org/10.1063/1.5032185
\end{abstract}

\section{INTRODUCTION}

Molecular triplet states represent an important outcome from excited state dynamics, and their presence can be detrimental as well as exploited. Consequently, understanding the mechanism of triplet formation is important for a broad range of molecular systems and not just in molecules containing heavier elements. In the simplest case, intersystem crossing (ISC) is driven by direct spin-orbit coupling (SOC) between two states of different multiplicity. These two states are considered in isolation, and the electronic spin-orbit coupling matrix elements (SOCMEs) are treated independently from the vibrational degrees of freedom, i.e., the Condon approximation. ${ }^{1}$ However, many recent time-resolved experiments, especially on transition metal systems, have illustrated that ISC is often not so straightforward. ${ }^{2,3}$ Indeed, ISC rates correlated to the vibrational period of important normal modes instead of the heavy atom effect ${ }^{4}$ or thermally activated intersystem crossing pathways, which depend upon specific molecular vibrations, ${ }^{5}$ have been observed. These results, which signify the breakdown of the Condon approximation, place an emphasis upon explicitly understanding the coupled dynamics of the spin, electronic, and vibrational components occurring within molecular excited states.

The communication between singlet and triplet states is especially important in the context of harvesting the triplet excited states generated upon electrical excitation in organic

a)Electronic mail: Julien.Eng@ncl.ac.uk.

b)Electronic mail: Tom.Penfold@ncl.ac.uk. light emitting diodes (OLEDs). The use of fluorescence emitters limits these devices, by spin-statistics, to a maximum internal quantum efficiency of $25 \%$, as $75 \%$ of the excitons are formed in non-radiative triplet states. Although this has been overcome using phosphorescence emitters, this approach relies upon molecules containing rare elements such as iridium and platinum. Recently, Thermally Activated Delayed Fluorescence (TADF) has emerged as a competitive approach and circumnavigates the reliance upon heavy elements. Here, the triplet states are harvested via the singlet states as delayed fluorescence using thermal energy. While a significant amount of the present focus in this area has been upon organic molecules, ${ }^{6-8}$ TADF has a strongly established history in $\mathrm{Cu}(\mathrm{I})$ complexes $^{9-11}$ and has also been reported in $\mathrm{Ag}(\mathrm{I})^{12}$ and $\mathrm{Au}(\mathrm{III})^{13,14}$ complexes.

In organic TADF molecules, the mechanism for efficient ISC and reverse ISC (rISC) has been demonstrated to be spinvibronic $^{15-19}$ and, consequently, depends upon specific vibrational degrees of freedom, which increases the mixing between singlet and triplet states. By contrast, for the transition metal TADF systems, the contribution of specific vibrational degrees of freedom has not received the same attention. Recently, Di et $a l .{ }^{20}$ proposed a cyclic (alkyl)(amino) carbene (CAAC) gold (I) complex (CMA1, Fig. 1), which exhibits efficient triplet harvesting and high performance when incorporated into an OLED device. The authors proposed that the triplet states are harvested by a so-called rotationally assisted spinstate inversion (RASI) mechanism. Here the rapid ISC and rISC were thought to arise because the $S_{1}$ state falls below the $\mathrm{T}_{1}$ state along the main reaction coordinate involving a torsion around the $\mathrm{Au}-\mathrm{N}_{2}$ bond ( $\varphi$, Fig. 1) creating an 


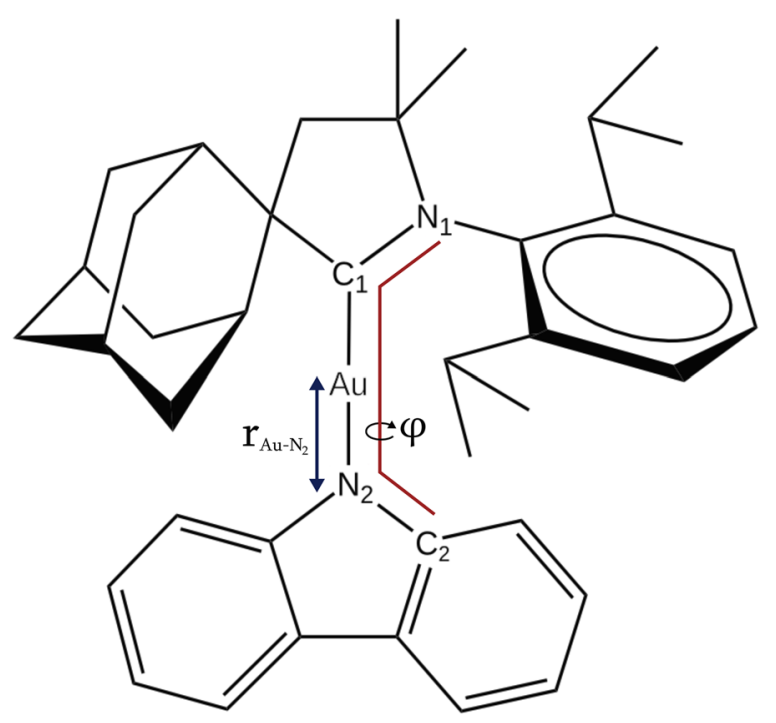

FIG. 1. Schematic of the cyclic (alkyl)(amino) carbene gold (I) complex (CMA1) studied herein. The stretching $\left(\mathrm{r}_{\mathrm{Au}-\mathrm{N}_{2}}\right)$ and torsional $(\varphi)$ degrees of freedom important in the quantum dynamics simulations are highlighted.

intersection between the states. This interpretation was supported by the observation that triplet harvesting was less effective in polycrystalline powders, which the authors proposed was due to the steric hindrance of $\varphi$. However, this mechanism is at odds with quantum mechanics, which states that a singlet and triplet state of the same character cannot cross. Indeed, within the one-electron limit, the two states are split by two times the exchange energy, which lowers the energy of the triplet state and lifts the energy of the singlet state. ${ }^{21}$

Recently Föller and Marian ${ }^{22}$ demonstrated that this proposed mechanism was founded upon the incorrect simultaneous use of unrestricted density functional theory (uDFT) and time-dependent density functional theory (TDDFT). Using quantum chemical calculations based upon the combined density functional theory and multireference configuration interaction (DFT/MRCI), ${ }^{23,24}$ they showed that ordering of the $S_{1}$ and $\mathrm{T}_{1}$ states does not invert along the torsional reaction coordinate. Instead it was proposed that the efficient equilibration between the $S_{1}$ and $T_{1}$ states was possible without invoking the RASI mechanism. The red shift in the luminescence spectrum of CMA1 in solution was assigned to a solvent reorganisation following a significant change in the dipole moment associated with excitation into a charge transfer (CT) state, i.e., the solvent adapting to the new electronic structure of excited CMA1.

This previous work highlights that the RASI mechanism cannot be correct; however, the rapid ISC and rISC reported in this complex and the high external quantum efficiencies for OLED devices mean that it represents an interesting material for such applications. In this present contribution, we use quantum dynamics simulations to provide detailed insight into the excited state dynamics of CMA1. By developing a model spin-vibronic Hamiltonian, we show that although previous calculations ${ }^{22}$ evaluated in the Condon approximation yield large ISC rates, our present dynamical treatment, taking into account the large amplitude torsional motion, increases this rate by an order of magnitude improving the agreement with experimental observations. Vibronic coupling to the higher lying triplet states appears to primarily alter the rISC process, although, in contrast to organic TADF systems, it is a detrimental effect due to the interference with the direct ISC pathways. The model spin-vibronic Hamiltonian developed is also general and consequently can straightforwardly be adapted to understand the properties of related linear metal carbene compounds, facilitating molecular design.

\section{METHODS}

\section{A. Quantum chemistry}

Geometry optimisations were performed using DFT and TDDFT for the ground and excited states, respectively, as implemented within the Q-Chem quantum chemistry package. $^{25}$ The PBE0 exchange and correlation functional ${ }^{26}$ was used, and the def2-SVP basis $\operatorname{set}^{27}$ was employed for all atoms. The corresponding electronic core potential (ECP) ${ }^{28}$ was used for gold. All optimisations were performed in the gas phase.

The excited state properties of CMA1 were calculated using TDDFT(PBE0) and the def2-TZVP basis set ${ }^{27}$ with the associated $\mathrm{ECP}^{28}$ for gold. The Tamm-Dancoff approximation (TDA ${ }^{29}$ was employed to avoid the over-stabilisation of low lying intra-ligand triplet states. The solvent has been modelled with the conductor-like screening model (COSMO) approach. ${ }^{30}$ Emission energies were calculated using the statespecific solvation approach as implemented within Q-Chem; i.e., both the fast (electronic) and slow (nuclear) components of the solvation model have been relaxed to accommodate the considered excited state. ${ }^{31}$ Spin-orbit coupling was computed using the ADF software suite ${ }^{32-34}$ using the zero order regular approximation (ZORA). ${ }^{35-37}$ These calculations were performed at the TDDFT(PBE0) level using a double- $\zeta$ polarized basis se $\mathrm{t}^{38-40}$ for hydrogens and a triple- $\zeta$ polarised basis set $^{38-40}$ for all other atoms.

\section{B. Spin-vibronic Hamiltonian and quantum dynamics}

To study the excited state intersystem crossing mechanism of CMA1, we adopt a 2-dimensional model spin-vibronic Hamiltonian. ${ }^{41-43}$ The present model spin-vibronic Hamiltonian includes the three lowest excited triplet states and the lowest excited singlet state $\left(\mathrm{S}_{1}\right)$. Each of the three $\mathrm{M}_{s}$ components has been explicitly included, meaning that the Hamiltonian contains 10 excited states.

The two nuclear degrees of freedom are the torsion around the Au- $\mathrm{N}_{2}$ bond $(\varphi)$ and the stretching mode $\left(\mathrm{r}_{\mathrm{Au}-\mathrm{N}_{2}}\right)$ of the same bond. Both motions are responsible for tuning the energy of the CT states. For the former, motion along this mode changes the relative energy gap between the $S_{1}$ and $T_{1}$ states, while motion along the latter $\left(\mathrm{r}_{\mathrm{Au}-\mathrm{N}_{2}}\right)$ modifies the energy gap between the CT states and the higher lying ligand centred excitations. While structural changes for the latter are small, the $1 / \mathrm{R}$ dependency of the energy of CT states with respect to the separation of the donor and acceptor groups (R) means it still plays an important role in the dynamics. Finally, we assume, 
consistent with experimental observations, that the initial geometric relaxation, occurring mostly in the CAAC ligand on the femtosecond time scale, plays a minimal role in the ISC which occurs on a much longer time scale, and therefore all dimensions not included in the Hamiltonian are at the minimum of $\mathrm{S}_{1}$ geometry.

The Hamiltonian operator is expressed as

$$
\mathcal{H}=\mathbf{T}_{\mathbf{N}}+\mathbf{W},
$$

where $\mathbf{T}_{\mathbf{N}}$ and $\mathbf{W}$ are the kinetic and potential energy operators, respectively, and are defined in Subsections II B 1-II B 3.

\section{The kinetic energy operator}

The form of the kinetic energy operator (KEO) depends on the coordinates chosen. Herein we adopt a KEO expressed as a sum of two uncoupled monodimensional KEOs,

$$
\mathbf{T}_{\mathbf{N}}=-\frac{1}{2 I} \frac{\partial^{2}}{\partial \varphi^{2}}-\frac{1}{2 \mu} \frac{\partial^{2}}{\partial r_{A u-N_{2}}^{2}} .
$$

The first term is the kinetic energy operator of the rotation of a solid top, where $I$ is the moment of inertia,

$$
I=\sum_{i} m_{i} r_{i}^{2},
$$

where $i$ denotes all atoms of the rotating carbazole ligand, $m_{i}$ denotes their mass, and $r_{i}$ denotes their distance to the rotation axis. The second term in the kinetic energy operator is associated with $\mathrm{r}_{\mathrm{Au}-\mathrm{N}_{2}}$, with $\mu$ being the reduced mass of the system,

$$
\mu=\frac{\left(\sum_{A} m_{A}\right)\left(\sum_{B} m_{B}\right)}{\sum_{A} m_{A}+\sum_{B} m_{B}},
$$

where $\mathrm{A}$ and $\mathrm{B}$ denote the atoms of the $\mathrm{Cz}$ moiety and of the CAAC ligand including the gold atom, respectively, and $m_{A}$, $m_{B}$ denote their respective mass. The parameters of the kinetic energy operator are $\mathrm{I}=3003.84 \mathrm{amu} \cdot \mathrm{a}_{0}^{2}$ and $\mu=128.91 \mathrm{amu}$. The implicit assumption in Eq. (2) is that the motion of $\varphi$ and $\mathrm{r}_{\mathrm{Au}-\mathrm{N}_{2}}$ are decoupled. This represents a valid approximation because $\mathrm{r}_{\mathrm{Au}-\mathrm{N}_{2}}$ is also the axis of rotation for $\varphi$. Consequently, the stretching motion does not change the distance of the $\mathrm{Cz}$ ligand atoms to the rotation axis, thus the moment of inertia $I$ is independent with respect to $\mathrm{r}_{\mathrm{Au}-\mathrm{N}_{2}}$.

\section{The potential}

The potential energy component of the Hamiltonian, $\mathbf{W}$, is expressed as

$$
\mathbf{W}=\mathbf{W}^{\mathbf{v i b}}+\mathbf{W}^{\text {soc }},
$$

where $\mathbf{W}^{\text {vib }}$ contains the diabatic electronic states and the coupling between them, while $\mathbf{W}^{\text {soc }}$ contains the SOCME in the diabatic picture. They are obtained by transformation of the computed SOCME between the so-called electronic adiabatic states $\left(\mathbf{V}^{\text {SOC }}\right)$. If $\mathcal{R}$ be the adiabatic to diabatic rotation matrix that transforms the adiabatic electronic states $\mathbf{V}$ into the diabatic electronic states matrix $\mathbf{W}^{\text {vib }}$ through the transformation

$$
\mathbf{W}^{\text {vib }}=\mathcal{R}^{-1} \mathbf{V} \mathcal{R} .
$$

$\mathbf{W}^{\text {soc }}$ is then obtained through the same rotation,

$$
\mathbf{W}^{\text {soc }}=\mathcal{R}^{-1} \mathbf{V}^{\text {soc }} \mathcal{R} .
$$

The vibronic coupling occurs between electronic states of the same spin multiplicity; $\mathbf{W}^{\text {vib }}$ can therefore be written as

$$
\mathbf{W}^{\mathrm{vib}}=\left(\begin{array}{cccr}
\mathbf{E}_{\mathbf{T}_{1}} & \lambda^{\mathbf{T} 1, \mathbf{T} 2} & \lambda^{\mathbf{T 1}, \mathbf{T 3}} & 0 \\
\lambda^{\mathbf{T 1}, \mathbf{T} 2} & \mathbf{E}_{\mathbf{T}_{2}} & \lambda^{\mathbf{T 2}, \mathbf{T 3}} & 0 \\
\lambda^{\mathbf{T 1}, \mathbf{T 3}} & \lambda^{\mathbf{T} 2, \mathbf{T 3}} & \mathbf{E}_{\mathbf{T}_{3}} & 0 \\
0 & 0 & 0 & \mathbf{E}_{\mathbf{S}_{1}}
\end{array}\right),
$$

with $E_{T_{n}}$ and $E_{S_{1}}$ being the diabatic potential energy for the triplet and $\mathrm{S}_{1}$ states, respectively, and $\lambda^{T n, T m}$ is the vibronic coupling between the $\mathrm{T}_{n}$ and $\mathrm{T}_{m}$ states. $\mathbf{W}^{\text {soc }}$ is written as

$$
\mathbf{W}^{\mathbf{s o c}}=\left(\begin{array}{cccc}
0 & \eta^{\mathbf{T} 1, \mathbf{T}_{2}} & \eta^{\mathbf{T}_{\mathbf{1}}, \mathbf{T}_{3}} & \eta^{\mathbf{T}_{1}, \mathbf{S}_{\mathbf{1}}} \\
\eta^{* \mathbf{T}_{1}, \mathbf{T}_{2}} & 0 & \eta^{\mathbf{T}_{2}, \mathbf{T}_{3}} & \eta^{\mathbf{T}_{2}, \mathbf{S}_{\mathbf{1}}} \\
\eta^{* \mathbf{T}_{1}, \mathbf{T}_{3}} & \eta^{* \mathbf{T}_{2}, \mathbf{T}_{3}} & 0 & \eta^{\mathbf{T}_{3}, \mathbf{S}_{1}} \\
\eta^{* \mathbf{T}_{1}, \mathbf{S}_{1}} & \eta^{* \mathbf{T}_{2}, \mathbf{S}_{1}} & \eta^{* \mathbf{T}_{3}, \mathbf{S}_{1}} & 0
\end{array}\right)
$$

with

$$
\begin{gathered}
\eta^{\mathbf{T}_{\mathbf{n}}, \mathbf{T}_{\mathbf{m}}}= \\
\left(\begin{array}{ccc}
\mathcal{I}\left(\eta^{\prime}\right) & -\mathcal{R}(\eta)+\mathcal{I}(\eta) & 0 \\
-\mathcal{R}(\eta)+\mathcal{I}(\eta) & 0 & \mathcal{R}(\eta)+\mathcal{I}(\eta) \\
0 & \mathcal{R}(\eta)+\mathcal{I}(\eta) & \mathcal{I}\left(\eta^{\prime}\right)
\end{array}\right)
\end{gathered}
$$

and

$$
\eta^{\mathbf{T}_{\mathbf{n}}, \mathbf{S}_{\mathbf{1}}}=\left(\begin{array}{c}
\mathcal{R}(\eta)+\mathcal{I}(\eta) \\
\mathcal{I}\left(\eta^{\prime}\right) \\
\mathcal{R}(\eta)-\mathcal{I}(\eta)
\end{array}\right),
$$

where $\mathcal{R}(\eta)$ and $\mathcal{I}(\eta)$ are the real and imaginary parts of the SOCMEs. In the present model, we consider them as constant, taking their value at the minimum of the $S_{1}$ excited state. The $3 \times 3$ matrix in Eq. (10) represents the coupling between each of the $\mathrm{M}_{s}$ components of the triplet states.

$\mathbf{W}^{\mathbf{v i b}}$ is constructed by a fit to the diabatic electronic states along the $\mathrm{r}_{\mathrm{Au}-\mathrm{N}_{2}}$ stretch and torsion, $\varphi$. The diabatic electronic states and coupling are computed using the diabatisation scheme ${ }^{44}$ implemented within Q-chem. The vibronic coupling obtained is quite weak, and therefore the diabatic states should be close to the adiabatic ones. The diabatic potential energy curves along the $\varphi$ degree of freedom are shown in Fig. 2(b). The potential energy surface (PES) of every states is quite flat, with the exceptions of the two energy barriers located around $\varphi=-30^{\circ}$ and $\varphi=155^{\circ}$, which arise from steric clashes between a hydrogen atom of the $\mathrm{Cz}$ ligand and a methyl group of the CAAC moiety. In the present model, they arise from the low dimensionality of our model spin-vibronic Hamiltonian. Indeed, in full nuclear configuration space, it is likely that thermal fluctuations of each coordinates will reduce the effect of this barrier. However, the elongation of the $\mathrm{r}_{\mathrm{Au}-\mathrm{N}_{2}}$ bond alone is not sufficient to prevent the sterical clash, and additional degrees of freedom are needed in order to do so 


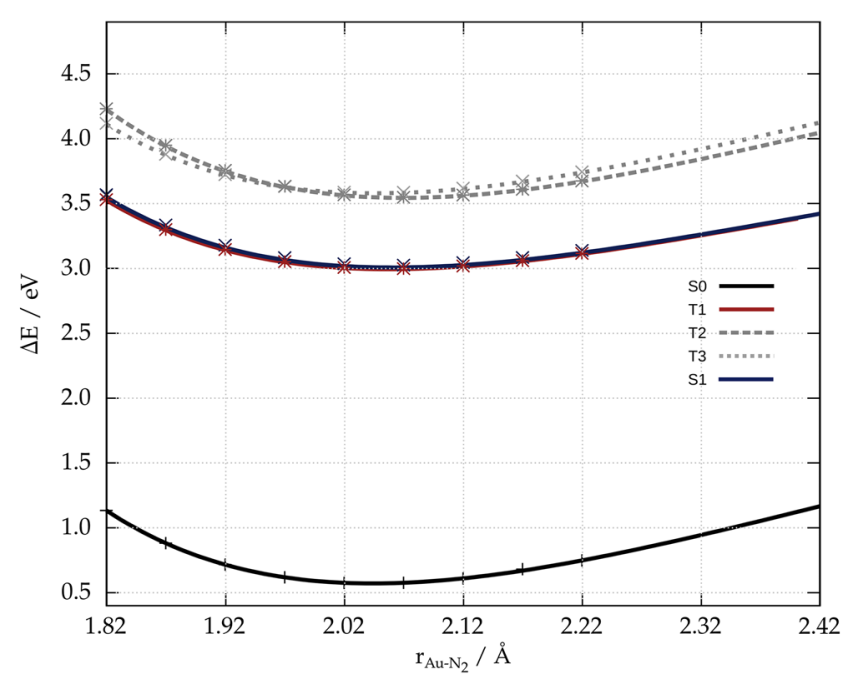

(a)

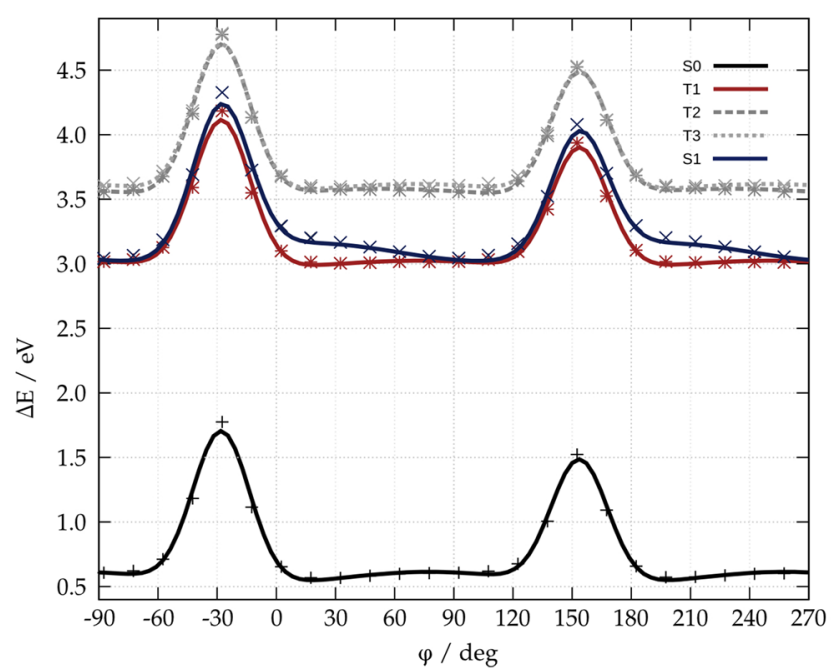

(b)

FIG. 2. Ground state and the four lowest excited diabatic states included in the model spin-vibronic Hamiltonian along (a) $\mathrm{r}_{\mathrm{Au}_{-} \mathrm{N}_{2}}$ and (b) $\varphi$.

and to allow free rotation around the $\mathrm{Au}-\mathrm{N}_{2}$ bond. The implications of this are discussed in the results. All states have been fitted by a sum of sine functions representing the torsion potential and two high order sine functions modeling the steric barriers,

$$
\begin{aligned}
E_{i}(\varphi)= & \beta_{i} \cos \left(2\left(\varphi+\tau_{i}\right)\right)+\gamma_{i} \cos \left(0.5\left(\varphi+\psi_{i}\right)\right)^{64} \\
& +\delta_{i} \cos \left(0.5\left(\varphi+\theta_{i}\right)\right)^{64}
\end{aligned}
$$

The parameters associated with the fits shown in Fig. 2 can be found in Table I.

The vibronic coupling along the torsion is shown in Fig. 3(b). The $T_{1}$ and $T_{2}$ states are coupled, as well as the $\mathrm{T}_{1}$ and $\mathrm{T}_{3}$ states. There is no vibronic coupling between $\mathrm{T}_{2}$ and $\mathrm{T}_{3}$ states. The vibronic coupling has been approximated using a sine function,

$$
\lambda^{T n, T m}(\varphi)=\epsilon \sin (\varphi+v),
$$

and the parameters of the fits shown in Fig. 3 are shown in Table I.
TABLE I. Parameters resulting from the fit of the diabatic states (top) and vibronic coupling (bottom) along the torsion $\varphi$.

\begin{tabular}{lrrrrr}
\hline \hline Parameter & \multicolumn{1}{c}{ GS } & \multicolumn{1}{c}{$T_{1}$} & $S_{1}$ & $T_{2}$ & \multicolumn{1}{c}{$T_{3}$} \\
\hline$\beta(\mathrm{eV})$ & -0.047 & -0.029 & 0.070 & 0.027 & -0.033 \\
$\gamma(\mathrm{eV})$ & 0.958 & 0.945 & 0.944 & 0.970 & 0.951 \\
$\delta(\mathrm{eV})$ & 1.174 & 1.158 & 1.161 & 1.184 & 1.166 \\
$\tau$ & 0.422 & 0.262 & -0.0311 & 0.548 & 0.290 \\
$\psi$ & 0.458 & 0.456 & 0.457 & 0.4555 & 0.456 \\
$\theta$ & 3.629 & 3.624 & 3.625 & 3.625 & 3.625 \\
\hline Parameter & $T_{1}-T_{2}$ & $T_{1}-T_{3}$ & & & \\
\hline$\epsilon(\mathrm{eV})$ & -0.059 & -0.073 & & & \\
$\nu$ & -1.546 & -1.676 & & & \\
\hline \hline
\end{tabular}

The diabatic states at the geometry of the minimum of $\mathrm{S}_{1}$ along the stretch coordinate $\mathrm{r}_{\mathrm{Au}-\mathrm{N}_{2}}$ are shown in Fig. 2, left. The Au- $\mathrm{N}_{2}$ bond length does not lift the degeneracy between $\mathrm{S}_{1}$ and $\mathrm{T}_{1}$. However $\mathrm{T}_{2}$ and $\mathrm{T}_{3}$ cross at small $\mathrm{r}_{\mathrm{Au}-\mathrm{N}_{2}}$ distances. The stretch potential is fitted using a Morse potential,

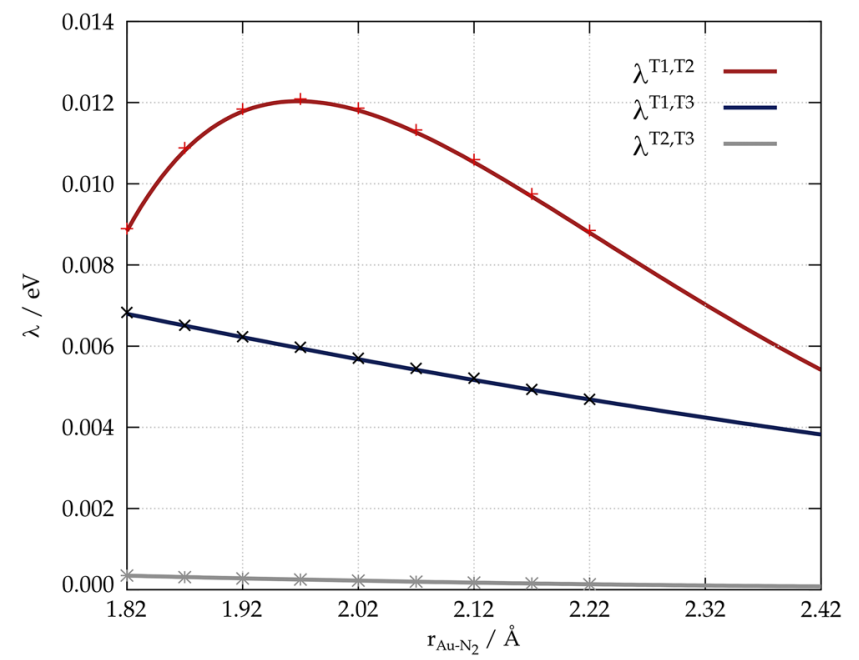

(a)

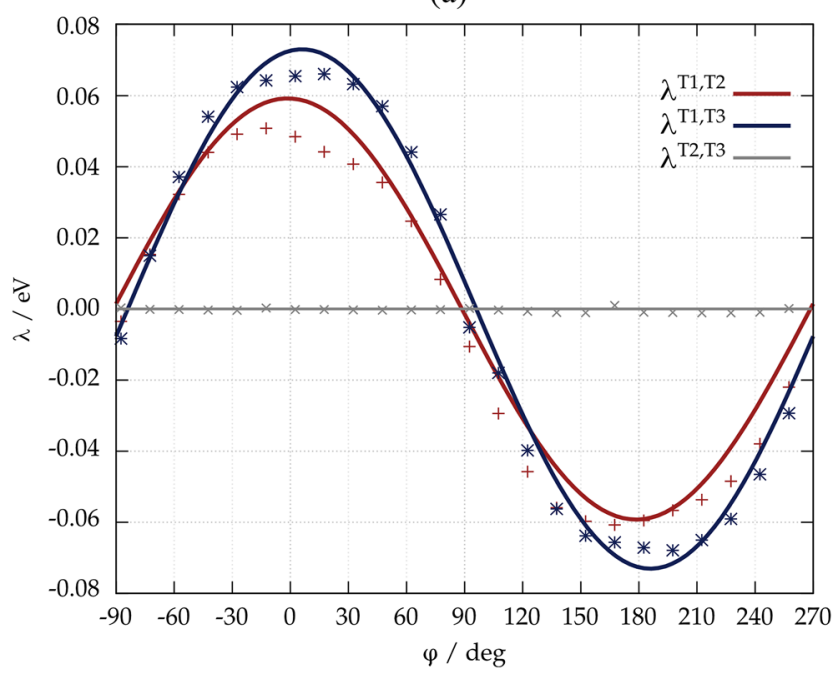

(b)

FIG. 3. Evolution of the vibronic coupling among the triplet manifold along (a) $\mathrm{r}_{\mathrm{Au}-\mathrm{N}_{2}}$ and (b) $\varphi$. 


$$
E_{i}\left(\mathrm{r}_{\mathrm{Au}-\mathrm{N}_{2}}\right)=D_{i}\left(\exp \left(-\alpha\left(\mathrm{r}_{\mathrm{Au}-\mathrm{N}_{2}}-\mathrm{r}_{\mathrm{Au}-\mathrm{N}_{2}}^{0}\right)\right)-1\right)^{2},
$$

where $D_{i}$ is the dissociation energy, $\alpha$ describes the width of the potential, and $\mathrm{r}_{\mathrm{Au}-\mathrm{N}_{2}}^{0}$ is the bond length at the minimum of energy. The parameters of the fit functions are shown in Table II.

The vibronic coupling along $\mathrm{r}_{\mathrm{Au}-\mathrm{N}_{2}}$ is shown in Fig. 3(a). A second order polynomial expression is sufficient to fit the coupling between $T_{1}$ and $T_{3}\left(\lambda^{T_{1}, T_{3}}\right)$ and between $T_{2}$ and $T_{3}$ $\left(\lambda^{T_{2}, T_{3}}\right)$,

$$
\lambda^{T_{n}, T_{m}}\left(\mathrm{r}_{\mathrm{Au}-\mathrm{N}_{2}}\right)=a \mathrm{r}_{\mathrm{Au}-\mathrm{N}_{2}}^{2}+b \mathrm{r}_{\mathrm{Au}-\mathrm{N}_{2}}+\lambda^{T_{n}, T_{m}}\left(\varphi_{0}\right),
$$

where $\lambda^{T_{n}, T_{m}}\left(\varphi_{0}\right)$ is the vibronic coupling at equilibrium geometry and is given by the coupling along the torsion $\varphi$. However, we need to use a Morse type function to fit properly the largest coupling $\lambda^{T_{1}, T_{2}}$,

$$
\begin{aligned}
\lambda^{T_{1}, T_{2}}\left(\mathrm{r}_{\mathrm{Au}-\mathrm{N}_{2}}\right)= & D_{i}\left(\exp \left(-\alpha\left(\mathrm{r}_{\mathrm{Au}-\mathrm{N}_{2}}-\mathrm{r}_{\mathrm{Au}-\mathrm{N}_{2}}^{0}\right)\right)-1\right)^{2} \\
& +\epsilon+\lambda^{T_{1}, T_{2}}\left(\varphi_{0}\right) .
\end{aligned}
$$

The parameters of the fit are shown in Table II.

\section{Wavepacket propagation}

The quantum dynamics were performed using the multiconfigurational time-dependent Hartree (MCTDH) method as implemented within the Quantics quantum dynamics package. ${ }^{45}$ The 2-dimensional model spin-vibronic Hamiltonian was represented on a Fast Fourier Transform (FFT) grid of $1001 \times 501$ grid points for the torsion and the stretching modes, respectively. The torsional mode was set with periodic boundary conditions ranging from $-\pi \rightarrow \pi$. The multi-set formalism was adopted, and 10 single particle functions (SPFs) were used for each mode on the $S_{1}$ and $T_{1}$ states and 4 SPFs were used for each mode on the $T_{2}$ and $T_{3}$ states. The evolution of the A-vector was calculated with the constant mean field integration scheme, and the SPFs were propagated with the Runge-Kutta integrator to eighth order (RK8). One initial wavepacket used in the simulations was obtained from a relaxation of a guessed wavepacket upon the ground state surface, vertically projected onto the $S_{1}$ surface. For the second set of simulations, a wavepacket with a larger width along the torsional mode was adopted to better represent the distribution

TABLE II. Parameters resulting from the fit of the diabatic states (top) and vibronic coupling (bottom) along the vibration $\mathrm{r}_{\mathrm{Au}-\mathrm{N}_{2}}$.

\begin{tabular}{lccccc}
\hline \hline Parameter & \multicolumn{1}{c}{$\mathrm{GS}$} & $\mathrm{T}_{1}$ & \multicolumn{1}{c}{$\mathrm{T}_{2}$} & \multicolumn{1}{c}{$\mathrm{T}_{3}$} & \multicolumn{1}{c}{$\mathrm{S}_{1}$} \\
\hline $\mathrm{D}_{\mathrm{i}}(\mathrm{eV})$ & 2.74 & 1.80 & 3.40 & 2.19 & 1.80 \\
$\alpha\left(\AA^{-1}\right)$ & 1.667 & 1.823 & 1.380 & 1.889 & 1.815 \\
$\mathrm{r}_{\mathrm{Au}-\mathrm{N}}(\AA)$ & 2.044 & 2.058 & 2.068 & 2.050 & 2.061 \\
$\epsilon_{i}(\mathrm{eV})$ & 0.00 & 2.42 & 2.97 & 3.01 & 2.44 \\
\hline Parameter & $\mathrm{T}_{1}-\mathrm{T}_{2}$ & & Parameter & $\mathrm{T}_{1}-\mathrm{T}_{3}$ & $\mathrm{~T}_{2}-\mathrm{T}_{3}$ \\
\hline $\mathrm{D}_{\mathrm{i}}(\mathrm{eV})$ & -0.0134 & & $a\left(\mathrm{eV} \AA^{-2}\right)$ & 0.0016 & 0.0004 \\
$\alpha\left(\AA^{-1}\right)$ & 2.6743 & & $b\left(\mathrm{eV} \AA^{-1}\right)$ & -0.0118 & -0.0023 \\
$\mathrm{r}_{\mathrm{Au}-\mathrm{N}}(\AA)$ & 1.9681 & & & & \\
$\epsilon_{i}(\mathrm{eV})$ & -0.0939 & & & & \\
\hline \hline
\end{tabular}

of $\varphi$ associated with the flat nature of the potential along this motion.

In the present spin-vibronic Hamiltonian, no decay channels to the ground state are incorporated and the Hamiltonian is therefore a closed quantum system. Consequently, at longer times, an equilibrium will form between the singlet and triplet states representing the balance between $k_{I S C}$ and $k_{r I S C}$. The population of the $S_{1}$ state, in this regime, can be expressed as ${ }^{19}$

$$
\left[S_{1}(t)\right]=1-\left[\frac{k_{I S C}}{k_{I S C}+k_{r I S C}} \times\left(1-\exp ^{-\left\{k_{I S C}+k_{I I S C}\right\} t}\right)\right],
$$

where $\left[S_{1}(t)\right]$ is the time-dependent population of the $S_{1}$ state. This equation is used to fit our population kinetics and extract $k_{I S C}$. It is noted that this approach can also be used to extract $\mathrm{k}_{r I S C}$. However, because our Hamiltonian has 2 dimensions, the energy which passes from the initial electronic excitation into the vibrational degrees of freedom, following decay from the $S_{1}$ into the $T_{1}$ state, cannot be dissipated. This makes the wavepacket in the $T_{1}$ state very vibrationally hot. $k_{r I S C}$ is temperature dependent, and so this high nuclear temperature will distort the rISC rate. Consequently, this term arising from our fits is not discussed throughout this work.

\section{RESULTS}

\section{A. Excited state properties}

The optimised ground-state geometry of CMA1 adopts the so-called co-planar conformation; i.e., the $\mathrm{Cz}$ and CAAC ligands are oriented in the same plane (Table III). This can be described using the $\varphi$ dihedral angle, shown in Fig. 1 . The ground state co-planar conformation is characterised by $\varphi=2.9^{\circ}$. At this geometry, the two lowest triplet excited states $\left(\mathrm{T}_{1}\right.$ and $\left.\mathrm{T}_{2}\right)$ are at $3.05 \mathrm{eV}$ and $3.16 \mathrm{eV}$. As shown in Table IV, these are both composed of a combination of $\mathrm{HOMO} \rightarrow \mathrm{LUMO}$ and $\mathrm{HOMO} \rightarrow \mathrm{LUMO}+1$ transitions. They

TABLE III. Energy of the co-planar and perpendicular conformations in $\mathrm{S}_{0}$, $\mathrm{S}_{1}$, and $\mathrm{T}_{1}$ and their characteristic values of $\mathrm{r}_{\mathrm{Au}-\mathrm{N}_{2}}$ and $\varphi$.

\begin{tabular}{lcc}
\hline \hline & Co-planar & Perpendicular \\
\hline$S_{0}$ & & \\
\hline$\Delta E(\mathrm{eV})$ & 0.00 & 0.11 \\
$\varphi(\mathrm{deg})$ & 2.9 & 87.1 \\
$\mathrm{r}_{\mathrm{Au}-\mathrm{N}_{2}}(\AA)$ & 2.05 & 2.06 \\
\hline$S_{1}$ & & \\
\hline$\Delta E(\mathrm{eV})$ & 3.01 & 2.94 \\
$\varphi(\mathrm{deg})$ & 22.4 & 89.8 \\
$\mathrm{r}_{\mathrm{Au}-\mathrm{N}_{2}}(\AA)$ & 2.11 & 2.08 \\
\hline$T_{1}$ & & 2.93 \\
\hline$\Delta E(\mathrm{eV})$ & 2.85 & 85.5 \\
$\varphi(\mathrm{deg})$ & 7.6 & 2.08 \\
$\mathrm{r}_{\mathrm{Au}-\mathrm{N}_{2}}(\AA)$ & 2.07 & \\
\hline \hline
\end{tabular}


TABLE IV. Electronic structure at the geometry of minimum energy in $\mathrm{S}_{0}$, $\mathrm{S}_{1}$, and $\mathrm{T}_{1}$. H represents the HOMO and L represents the LUMO.

\begin{tabular}{lcccc}
\hline \hline \multicolumn{4}{l}{$\mathrm{S}_{0}$ minimum } \\
\hline State & Excitation & Weight $(\%)$ & Osc. str. & Energy (eV) \\
\hline$T_{1}$ & $H \rightarrow L$ & 55 & 0.000 & 3.05 \\
& $H \rightarrow L+1$ & 40 & & \\
\hline$T_{2}$ & $H \rightarrow L$ & 41 & 0.000 & 3.16 \\
& $H \rightarrow L+1$ & 55 & & \\
\hline$S_{1}$ & $H \rightarrow L$ & 99 & 0.178 & 3.30 \\
$T_{3}$ & $H-1 \rightarrow L+1$ & 77 & 0.000 & 3.40 \\
\hline
\end{tabular}

$\mathrm{S}_{1}$ minimum

\begin{tabular}{lcccc}
\hline State & Excitation & Weight $(\%)$ & Osc. str. & Energy (eV) \\
\hline$S_{0}$ & $\ldots$ & $\ldots$ & $\ldots$ & 0.56 \\
$T_{1}$ & $H \rightarrow L$ & 99 & 0.000 & 2.97 \\
$S_{1}$ & $H \rightarrow L$ & 99 & 0.001 & 2.98 \\
$T_{2}$ & $H \rightarrow L+1$ & 81 & 0.000 & 3.51 \\
$T_{3}$ & $H-2 \rightarrow L$ & 95 & 0.000 & 3.56 \\
\hline
\end{tabular}

$\mathrm{T}_{1}$ minimum

\begin{tabular}{lcccc}
\hline State & Excitation & Weight $(\%)$ & Osc. str. & Energy $(\mathrm{eV})$ \\
\hline$S_{0}$ & $\ldots$ & $\ldots$ & $\ldots$ & 0.35 \\
$T_{1}$ & $H \rightarrow L$ & 97 & 0.000 & 2.89 \\
$S_{1}$ & $H \rightarrow L$ & 99 & 0.162 & 3.11 \\
$T_{2}$ & $H \rightarrow L+1$ & 96 & 0.000 & 3.40 \\
$T_{3}$ & $H-2 \rightarrow L$ & 90 & 0.000 & 3.57 \\
\hline \hline
\end{tabular}

are therefore a mix of charge transfer from the $\mathrm{Cz}$ to the CAAC ligand and a local excitation on the $\mathrm{Cz}$ ligand. The third triplet state, $\mathrm{T}_{3}$, is at $3.40 \mathrm{eV}$ and is a local excitation on the $\mathrm{Cz}$ ligand. The absorbing singlet state $S_{1}$ falls between the $T_{2}$ and $T_{3}$ states, at $3.30 \mathrm{eV}$, and is a pure HOMO $\rightarrow$ LUMO transition, i.e., a charge transfer state from the $\mathrm{Cz}$ to the CAAC ligand. The $\mathrm{S}_{0} \rightarrow \mathrm{S}_{1}$ absorption of $3.30 \mathrm{eV}$ agrees well with the first peak in the experimental absorption spectrum $(\sim 364 \mathrm{~nm})$ and recent calculations, at a higher level of theory by Föller and Marian. ${ }^{22}$ The electronic difference densities associated with each of these transitions are shown in Fig. 4.

The co-planar arrangement is not the only minimum on the ground state potential energy surface (PES), and the perpendicular conformation can also be optimised. The energy difference between the two conformers is only $0.11 \mathrm{eV}$, highlighting the shallow nature of the potential along the torsional mode of the $\mathrm{Cz}(\varphi)$. To minimise steric hindrance between a hydrogen atom of the $\mathrm{Cz}$ ligand and a methyl group of the CAAC moiety, the $\mathrm{Au}-\mathrm{N}_{2}$ bond length is longer than at the co-planar minimum, $\mathrm{r}_{\mathrm{Au}-\mathrm{N}_{2}}=2.06 \AA$ versus $\mathrm{r}_{\mathrm{Au}-\mathrm{N}_{2}}=2.05 \AA$. The electronic structure for the perpendicular conformation is similar to the one at the co-planar geometry.

The $T_{1}$ and $S_{1}$ potential energy surfaces (PESs) exhibit a similar topology with one minimum at the co-planar geometry $\left(\varphi=22.4^{\circ}\right.$ in $\mathrm{S}_{1}, \varphi=7.6^{\circ}$ in $\left.\mathrm{T}_{1}\right)$ and another one at a perpendicular conformation $\left(\varphi=89.8^{\circ}\right.$ in $S_{1}, \varphi=85.5^{\circ}$ in $\mathrm{T}_{1}$ ). The perpendicular conformation is the most stable in $\mathrm{S}_{1}$, whereas the co-planar conformation is more favourable in $\mathrm{T}_{1}$. An elongation of the $\mathrm{Au}-\mathrm{N}_{2}$ bond is observed in the co-planar minimum of $\mathrm{S}_{1}$, from $\mathrm{r}_{\mathrm{Au}-\mathrm{N}_{2}}=2.05 \AA$ in the ground state to $\mathrm{r}_{\mathrm{Au}-\mathrm{N}_{2}}=2.11 \AA$ in $\mathrm{S}_{1}$. This reduces the HOMO/LUMO overlap and thus the exchange energy between the electrons. This effect is not observed for the perpendicular conformation as the relative orientation of the two ligands already ensures a zero overlap. The exchange energy lowers the energy of the triplet states; therefore, the bond elongation is not observed in $T_{1}$. This explains partly why the most stable conformation in $S_{1}$ is the perpendicular conformation, while the most favourable one in $\mathrm{T}_{1}$ is the co-planar form. At $\mathrm{S}_{1}$ co-planar minimum, the $T_{1}$ and $S_{1}$ states are nearly degenerate $\left(\Delta E^{S_{1}, T_{1}}=0.01 \mathrm{eV}\right)$ and both consist of pure HOMO $\rightarrow$ LUMO transitions. The $\mathrm{T}_{2}$ is at $3.40 \mathrm{eV}$ and, in contrast to the ground state geometry, is now a pure $\mathrm{HOMO}$ to LUMO+1 transition. At this geometry, $\mathrm{T}_{3}$ is no longer a local excitation on the $\mathrm{Cz}$ ligand but is now a local excitation on the CAAC. The difference of electronic density associated with these transitions is shown in Fig. 5. At
$\mathrm{T}_{1}$
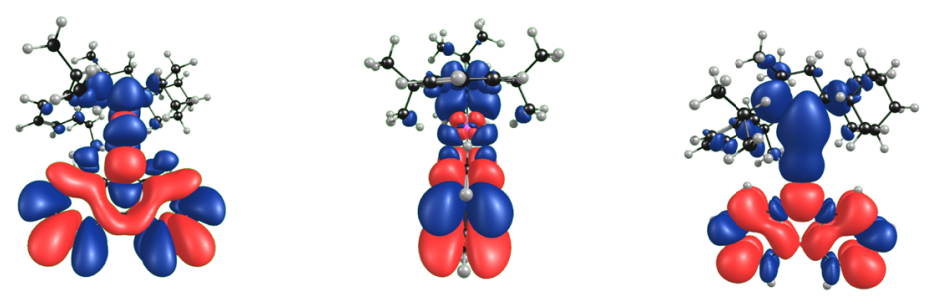

$\mathrm{S}_{1}$

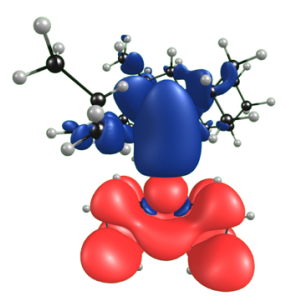

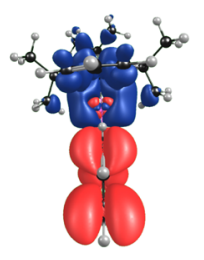

$\mathrm{T}_{2}$

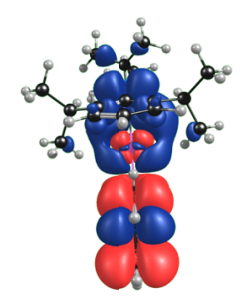

$\mathrm{T}_{3}$

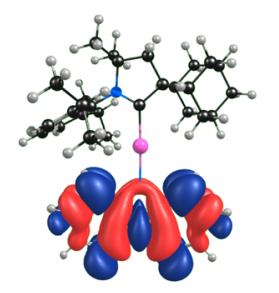

FIG. 4. Difference of electronic density associated with the electronic states $\mathrm{T}_{1}, \mathrm{~T}_{2}, \mathrm{~S}_{1}$, and $\mathrm{T}_{3}$ at the geometry of minimum energy in the ground state of CMA1. For each state, front (left) and side (right) views are shown. Loss of electronic density is shown in red; gain is shown in blue. 
$\mathrm{T}_{1}$
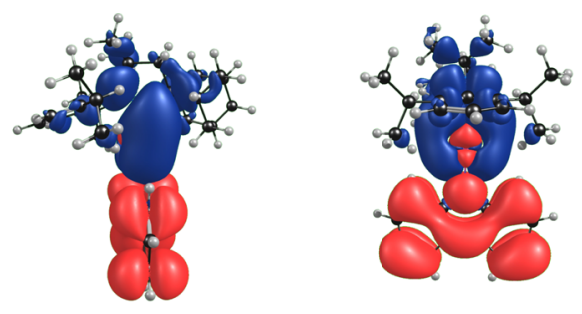

$\mathrm{T}_{2}$

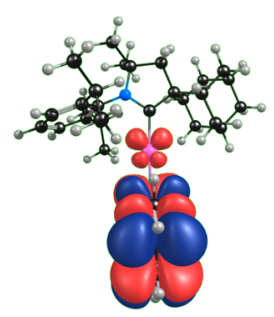

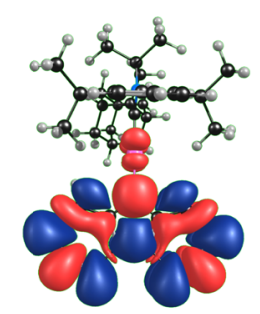

$\mathrm{S}_{1}$

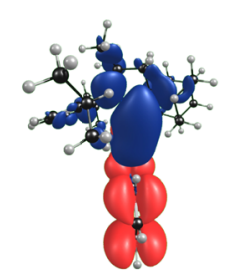

$T_{3}$

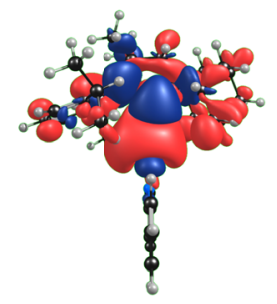

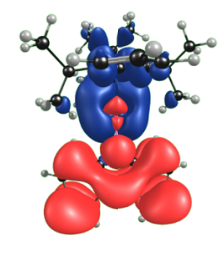

FIG. 5. Difference of electronic density associated with the electronic states $\mathrm{T}_{1}, \mathrm{~T}_{2}, \mathrm{~S}_{1}$, and $\mathrm{T}_{3}$ at the geometry of minimum energy of $S_{1}$ of CMA1. For each state, front (left) and side (right) views are shown. Loss of electronic density is shown in red; gain is shown in blue. the $S_{1}$ optimised geometry, the $S_{1}$ energy is $2.97 \mathrm{eV}(417 \mathrm{~nm})$, meaning a Stokes shift of $0.34 \mathrm{eV}$, which is primarily from the stabilisation of the CAAC ligand, lowering the energy of the LUMO and therefore closing the HOMO-LUMO gap. This exhibits little agreement with the experimental emission spectrum; however, as described by Föller and Marian, ${ }^{22}$ this deviation can be accounted for by including the effect of the excited state solvent rearrangement. Using a state-specific continuum model, we find an emission energy of $2.20 \mathrm{eV}(563.5 \mathrm{~nm})$ for toluene, in very good agreement with the emission reported experimentally.

In terms of the SOCME, important for coupling the singlet and triplet manifolds, at the $S_{1}$ geometry, the $T_{1}$ and $S_{1}$ states are of the same character and therefore exhibit a relatively small spin-orbit coupling of $\eta^{S_{1}, T_{1}}=13.87 \mathrm{~cm}^{-1}$. In contrast the $\mathrm{T}_{2}$ state, which consists of a pure local exciton on the CAAC ligand, involves a d orbital of the gold metal. This promotes strong SOC between the $T_{1}$ and $T_{2}$ and the $S_{1}$ and $\mathrm{T}_{2}$ states of $\eta^{T_{1}, T_{2}}=1393.69 \mathrm{~cm}^{-1}$ and $\eta^{S_{1}, T_{2}}=649.22 \mathrm{~cm}^{-1}$, respectively. The SOCMEs between the low lying states and $\mathrm{T}_{3}$ are $\eta^{T_{1}, T_{3}}=15.30 \mathrm{~cm}^{-1}, \eta^{T_{2}, T_{3}}=26.57 \mathrm{~cm}^{-1}$, and $\eta^{S_{1}, T_{3}}=59.09 \mathrm{~cm}^{-1}$.

\section{B. Quantum dynamics}

In this section, we use quantum dynamics in combination with the spin-vibronic Hamiltonian, developed in Sec. II B, to study the ISC dynamics after excitation into the $S_{1}$ state. The dynamics are initiated from an initial wavepacket obtained from a relaxation of the wavefunction upon the ground state surface and wider initial wavepacket to account for the distribution in $\varphi$ expected at room temperature.

Figure 6 shows the $S_{1}$ and $T_{1}$ population kinetics during the first $100 \mathrm{ps}$ after photoexcitation into the $S_{1}$ state when adopting the narrow wavefunction, i.e., the wavefunction obtained from the relaxation. A steady decay of $S_{1}$ into the $T_{1}$ state is observed throughout the $100 \mathrm{ps}$ of dynamics. At early times, these kinetics are characterised by large amplitude oscillations, which are associated with the initial narrow wavepacket moving along the $\varphi$ degree of freedom and reaching the point of degeneracy between two states. As described in Sec. II, we use Eq. (17) to extract $\mathrm{k}_{I S C}$ and find a value of $2.20 \times 10^{10} \mathrm{~s}^{-1}$. The dotted light blue line in Fig. 6 shows the same dynamics, but in this case the steric barrier shown in Fig. 2 has been removed, allowing free rotation of the $\varphi$ torsion. This has the effect of slowing the ISC dynamics

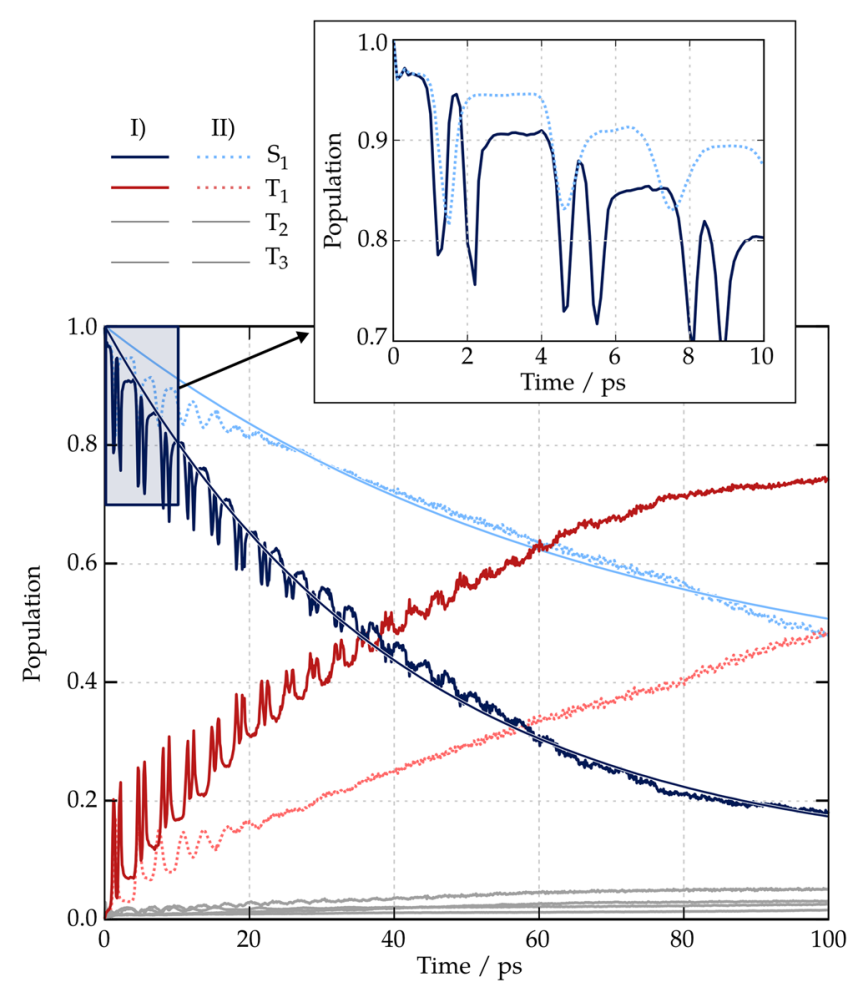

FIG. 6. Evolution of the excited state populations, $\mathrm{S}_{1}$ (blue) and $\mathrm{T}_{1}$ (red), with time. Initial wavepacket obtained from a relaxation of the wavefunction on the ground state surface. Full lines (I): Dynamics using the spin-vibronic Hamiltonian described in Sec. II B. Dotted lines (II): Dynamics with no energy barrier along the torsion $\varphi$. 
slightly, although $\mathrm{k}_{I S C}=9.53 \times 10^{9} \mathrm{~s}^{-1}$ remains within a factor of 3 .

The population transfer kinetics from $S_{1}$ to $T_{1}$ exhibits plateaux (Fig. 6, inset). These features are indicative of the fact that the population is transferred principally in the region of near degeneracy (i.e., perpendicular geometry) between $\mathrm{T}_{1}$ and $\mathrm{S}_{1}$, resulting in "peaks" in the population kinetics. This region is reached for the first time around $t=1250 \mathrm{fs}$ in the presence of steric barriers and at $t=1460 \mathrm{fs}$ for the free rotation. This variation arises from the difference in the gradient of the potential in the vicinity of the co-planar conformation. The wavepacket then continues evolving along the torsion $\varphi$. In the absence of steric barriers, the periodic torsional coordinates allows free rotation and the wavepacket eventually reaches another region of near degeneracy leading to the next significant transfer of population from $S_{1}$ to $T_{1}$. The presence of steric barriers prevents such free rotation, and the wavepacket is pushed back and returns to the near degeneracy region leading to the double peak structure. This description

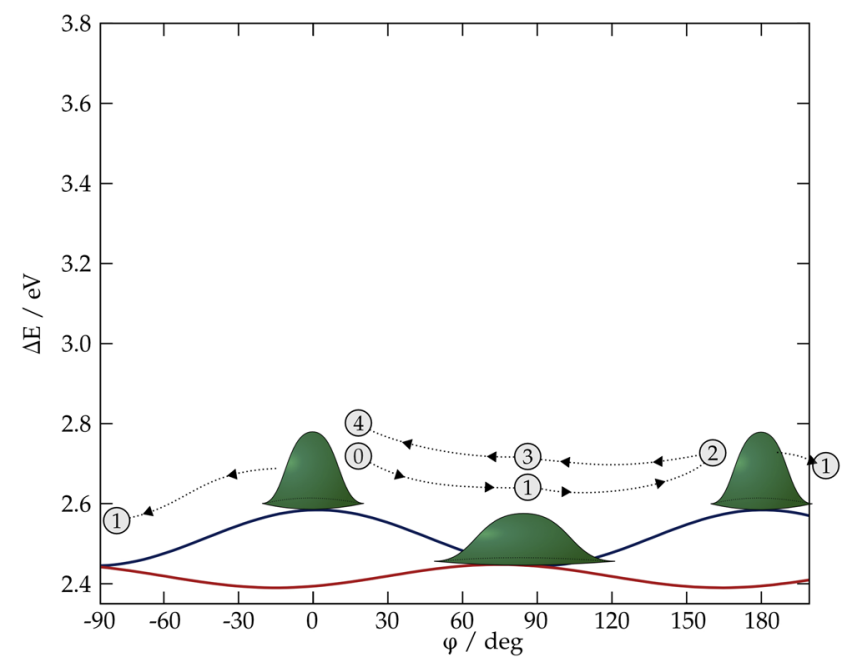

(a)

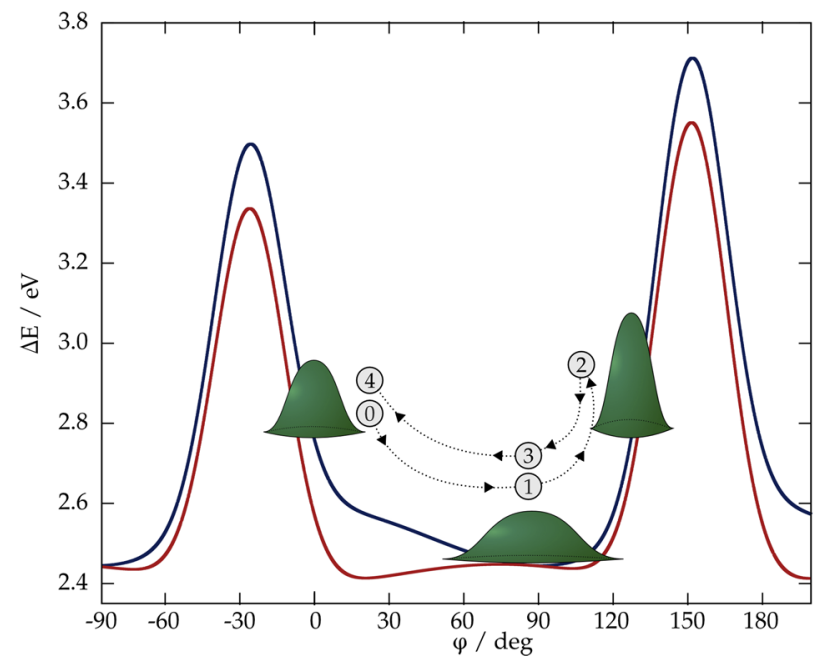

(b)

FIG. 7. Schematic representation of the effect of the sterical barrier on the dynamics of the molecular wavepacket (a) in the case of free rotation and (b) in the presence of sterical barriers. Snapshots of the wavepacket at different times are shown with labels from 0 to 4 . is schematically shown in Fig. 7. The frequency of passing of the wavepacket through the near degeneracy region is $v_{\text {barrier }}=2.94 \times 10^{11} \mathrm{~Hz}$ in the presence of steric barriers and $v_{\text {barrierless }}=1.65 \times 10^{11} \mathrm{~Hz}$ in their absence. The factor 1.8 between those two frequencies partly explains the difference of $k_{I S C}$ between both simulations. In addition to this effect, the wavepacket lies close to the near degeneracy region when colliding with the steric barrier, allowing for additional population transfer.

Figure 8 shows the $S_{1}$ and $T_{1}$ population kinetics during the first 100 ps when adopting a wider initial wavepacket. As expected, its largest effect is to damp the large amplitude changes associated with a localised wavepacket meeting the point of degeneracy. This wider wavepacket mimics the large distribution of the $\varphi$ angle in the ground state expected at room temperature induced by the flat electronic potential of the ground state along the torsion. When reduced, the kinetics still exhibit step changes, which are associated with the simplicity of the 2-dimensional spin-vibronic Hamiltonian, which restricts the flow of vibrational energy to those modes, rather than all vibrational degrees of freedom of the molecule and the solvent, which would provide decoherence. The wider wavepacket only slightly speeds up the rate of intersystem crossing as the wavepacket no longer has to traverse the $\varphi$ coordinate to undergo ISC. Here we find $\mathrm{k}_{I S C}=2.76 \times 10^{10} \mathrm{~s}^{-1}$, and if the steric barrier is removed, $\mathrm{k}_{I S C}=1.37 \times 10^{10} \mathrm{~s}^{-1}$. While both of these are in good agreement with the rates reported experimentally, they also demonstrate that the initial choice of wavepacket does not fundamentally alter the interpretation presented herein.

Importantly, in terms of the mechanism of ISC, Fig. 8 also shows the population kinetics for which the coupling with $T_{2}$ and $T_{3}$ states has been removed. Here we observe a rate of population transfer from $S_{1}$ to $T_{1}$ of $3.78 \times 10^{11} \mathrm{~s}^{-1}$, in

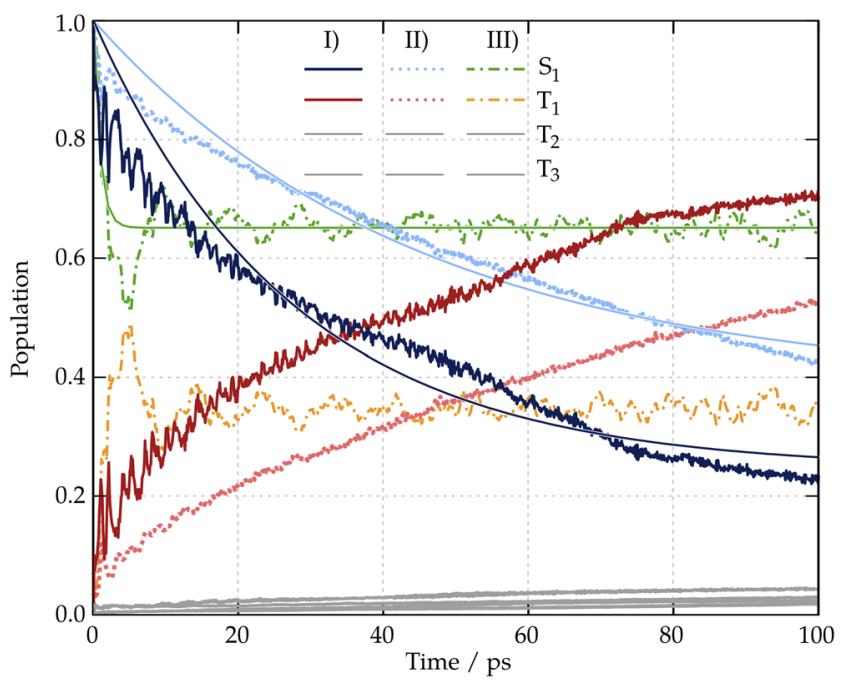

FIG. 8. Evolution of the excited state populations, $\mathrm{S}_{1}$ (blue) and $\mathrm{T}_{1}$ (red), with time. A wider initial wavepacket is used to account for the distribution in $\varphi$ due to the flat potential energy along the torsion $\varphi$. Full lines (I): Dynamics using the spin-vibronic Hamiltonian described in Sec. II B. Dotted lines (II): Dynamics with no energy barrier along the torsion $\varphi$. Dashed-dotted lines (III): Dynamics without $\mathrm{T}_{2}$ nor $\mathrm{T}_{3}$. For clarity purpose, $\mathrm{S}_{1}$ is shown in green and $\mathrm{T}_{1}$ is shown in orange. 


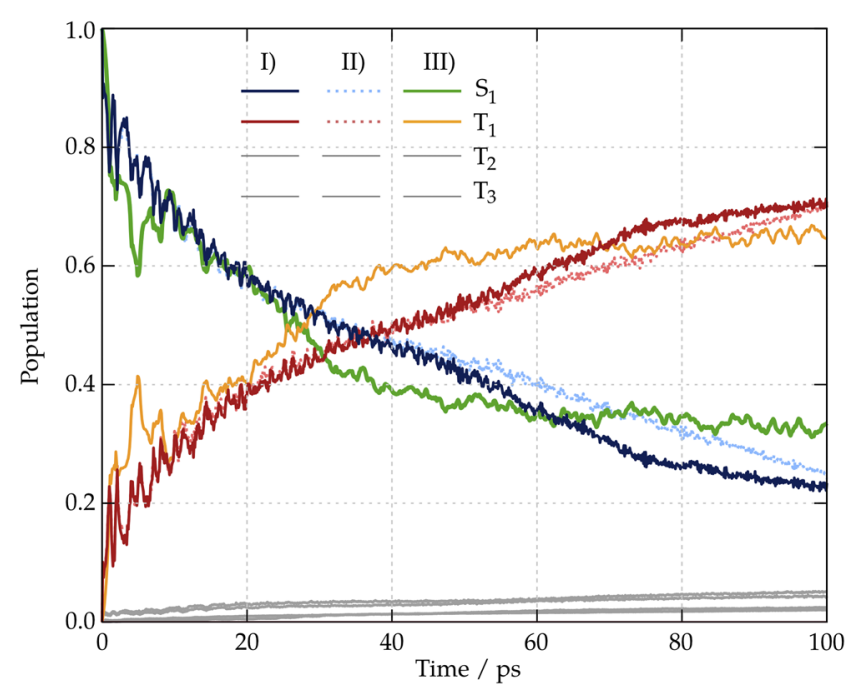

FIG. 9. Evolution of the excited state populations, $S_{1}$ (blue) and $T_{1}$ (red), with time. (I) Dynamics using the spin-vibronic Hamiltonian described in Sec. II B. (II) Dynamics using the same Hamiltonian without any coupling to $T_{2}$. (III) Dynamics using the same Hamiltonian without any coupling to $\mathrm{T}_{3}$. For the purpose of clarity, $\mathrm{S}_{1}$ is shown in green and $T_{1}$ is shown in orange.

close agreement with the rates observed experimentally and an order of magnitude larger than for the full spin-vibronic Hamiltonian. In addition, a plateau in the population transfer is reached within $20 \mathrm{ps}$, indicating equilibration between the $\mathrm{S}_{1}$ and $\mathrm{T}_{1}$ states and therefore a faster $k_{r I S C}$ than for the full Hamiltonian. Figure 9 shows the effect of individual triplet states on the relaxation dynamics. We successively remove each triplet from the Hamiltonian. The absence of $\mathrm{T}_{2}$ [Fig. 9(II)] or $\mathrm{T}_{3}$ [Fig. 9(III)] does not prevent the transfer of population from $S_{1}$ to $T_{1}$, and the relaxation rate is similar to the one obtained from the full Hamiltonian simulation [Fig. 9(I)].

These simulations show that the presence of the $T_{2}$ and $\mathrm{T}_{3}$ modulates the equilibrium ratio of the population in $\mathrm{S}_{1}$ and $T_{1}$ and is therefore indicative of a change in the ratio between the ISC and the rISC rates. Indeed, the presence of at least one of the higher lying states actually suppresses $k_{\text {rISC }}$ because equilibrium between the two states is not reached within 100 ps of dynamics. This is in contrast to the observation made for organic TADF systems. This would appear to suggest the role of inferences between the direct $\left(S_{1}-T_{1}\right)$ and spin-vibronic pathways, i.e., either $T_{1}-T_{2}-S_{1}$ or $T_{1}-T_{3}-S_{1}$ pathways. These interferences increase ISC from $10^{10} \mathrm{~s}^{-1}$ to $10^{11} \mathrm{~s}^{-1}$ and provide excellent agreement with the experimentally reported rate of $\mathrm{k}_{\mathrm{ISC}}=2.5 \times 10^{11} \mathrm{~s}^{-120}$ but importantly decreases the $k_{r I S C}$, which is undesirable for high performing OLED devices.

\section{CONCLUSIONS}

The present study has used quantum chemistry and quantum dynamics to shed new insight into the excited state properties and intersystem crossing mechanism of CMA1. In agreement with recent work, ${ }^{22}$ our quantum chemistry confirms that the proposed RASI mechanism ${ }^{20}$ is not correct and the lowest singlet and triplet states do not cross.
The quantum dynamics shows that the ISC dynamics occurs on the time scale of tens of picosecond and along the torsional mode, allowing the wavepacket to reach regions of the potential where the singlet and triplet states are degenerate. It is noted that ISC is possible all along the torsional mode but is fastest at the point of degeneracy. Importantly, only a $\sim 30^{\circ}$ change in the dihedral is required to get close to the point of degeneracy and therefore using molecular dynamics to observe how much this motion is restricted in the solid state will be important for understanding the operation of CMA1 in an OLED device.

Our dynamics reveal a spin-vibronic component within the present model with higher lying triplet states. In this case, they act as virtual states in the sense that they contribute to the dynamics, without receiving significant population themselves. This has the strongest influence on $k_{r I S C}$ and is indeed expected to slow it down. This arises from interference effects between direct $\left(S_{1}-T_{1}\right)$ ISC and spin-vibronic pathways, i.e., $T_{1}-T_{2}-S_{1}$ or $T_{1}-T_{3}-S_{1}$; and the observed behavior is in contrast to organic TADF systems ${ }^{16-19}$ as in these cases the direct ISC pathway is very weak.

In summary, this work has provided further insight into the excited state dynamics of CMA1. Importantly, the spin-vibronic Hamiltonian developed can be extended to the analogous $\mathrm{Cu}$ and $\mathrm{Ag}$ systems, which is presently underway.

\section{ACKNOWLEDGMENTS}

We acknowledge the EPSRC, Project No. EP/P012167/1, funding. Data supporting this publication are openly available under an "Open Data Commons Open Database License." Additional meta-data are available at 10.17634/162036-1. Please contact Newcastle Research Data Service at rdm@ncl.ac.uk for access instructions.

${ }^{1}$ C. M. Marian, Wiley Interdiscip. Rev.: Comput. Mol. Sci. 2, 187 (2012).

${ }^{2}$ M. Chergui, Dalton Trans. 41, 13022 (2012).

${ }^{3}$ T. J. Penfold, E. Gindensperger, C. Daniel, and C. M. Marian, "Spin-vibronic mechanism for intersystem crossing," Chem. Rev. (published online).

${ }^{4}$ A. Cannizzo, A. M. Blanco-Rodriguez, A. El Nahhas, J. Šebera, S. Záliš, A. Vlček, and M. Chergui, J. Am. Chem. Soc. 130, 8967 (2008).

${ }^{5}$ T. Hofbeck, Y. C. Lam, M. Kalbáč, S. Záliš, A. Vlček, and H. Yersin, Inorg. Chem. 55, 2441 (2016).

${ }^{6}$ H. Uoyama, K. Goushi, K. Shizu, H. Nomura, and C. Adachi, Nature 492, 234 (2012).

${ }^{7}$ F. B. Dias, T. J. Penfold, and A. P. Monkman, Methods Appl. Fluoresc. 5, 012001 (2017).

${ }^{8}$ T. J. Penfold, F. B. Dias, and A. P. Monkman, Chem. Commun. 54, 3926 (2018).

${ }^{9}$ J. R. Kirchhoff, R. E. Gamache, Jr., M. W. Blaskie, A. A. Del Paggio, R. K. Lengel, and D. R. McMillin, Inorg. Chem. 22, 2380 (1983).

${ }^{10}$ J. C. Deaton, S. C. Switalski, D. Y. Kondakov, R. H. Young, T. D. Pawlik, D. J. Giesen, S. B. Harkins, A. J. M. Miller, S. F. Mickenberg, and J. C. Peters, J. Am. Chem. Soc. 132, 9499 (2010).

${ }^{11}$ R. Czerwieniec, M. J. Leitl, H. H. H. Homeier, and H. Yersin, Coord. Chem. Rev. 325, 2 (2016).

${ }^{12}$ M. Z. Shafikov, A. F. Suleymanova, R. Czerwieniec, and H. Yersin, Chem. Mater. 29, 1708 (2017).

${ }^{13}$ W.-P. To, D. Zhou, G. So Ming Tong, G. Cheng, C. Yang, and C.-M. Che, Angew. Chem., Int. Ed. 129, 14224 (2017).

${ }^{14}$ J. Fernandez-Cestau, B. Bertrand, M. Blaya, G. A. Jones, T. J. Penfold, and M. Bochmann, Chem. Commun. 51, 16629 (2015).

${ }^{15}$ J. Gibson, A. P. Monkman, and T. J. Penfold, ChemPhysChem 17, 2956 (2016). 
${ }^{16}$ M. K. Etherington, J. Gibson, H. F. Higginbotham, T. J. Penfold, and A. P. Monkman, Nat. Commun. 7, 13680 (2016).

${ }^{17}$ M. K. Etherington, F. Franchello, J. Gibson, T. Northey, J. Santos, J. S. Ward, H. F. Higginbotham, P. Data, A. Kurowska, P. Lays Dos Santos et al., Nat. Commun. 8, 14987 (2017).

${ }^{18}$ C. M. Marian, J. Phys. Chem. C 120, 3715 (2016).

${ }^{19}$ I. Lyskov and C. M. Marian, J. Phys. Chem. C 121, 21145 (2017).

${ }^{20}$ D. Di, A. S. Romanov, Le Yang, J. M. Richter, J. P. H. Rivett, S. Jones, T. H. Thomas, M. A. Jalebi, R. H. Friend, M. Linnolahti et al., "Highperformance light-emitting diodes based on carbene-metal-amides," Science 356, 159 (2017).

${ }^{21}$ N. J. Turro, Modern Molecular Photochemistry (University Science Books, 1991).

${ }^{22}$ J. Fóller and C. M. Marian, J. Phys. Chem. Lett. 8, 5643 (2017).

${ }^{23}$ S. Grimme and M. Waletzke, J. Chem. Phys. 111, 5645 (1999).

${ }^{24}$ I. Lyskov, M. Kleinschmidt, and C. M. Marian, J. Chem. Phys. 144, 034104 (2016).

${ }^{25}$ Y. Shao et al., Mol. Phys. 113, 184 (2015).

${ }^{26} \mathrm{C}$. Adamo and V. Barone, J. Chem. Phys. 110, 6158 (1999).

${ }^{27}$ F. Weigend and R. Ahlrichs, Phys. Chem. Chem. Phys. 7, 3297 (2005).

${ }^{28}$ D. Andrae, U. Häußermann, M. Dolg, H. Stoll, and H. Preuß, Theor. Chem. Acc. 77, 123 (1990).

${ }^{29}$ S. Hirata and M. Head-Gordon, Chem. Phys. Lett. 314, 291 (1999).

${ }^{30}$ A. Klamt and G. J. Schüürmann, J. Chem. Soc., Perkin Trans. 2 5, 799-805 (1993).

${ }^{31}$ A. Klamt, J. Phys. Chem. 100, 3349 (1996).
${ }^{32}$ G. Te Velde, F. M. Bickelhaupt, E. J. Baerends, C. Fonseca Guerra, S. J. A. van Gisbergen, J. G. Snijders, and T. Ziegler, J. Comput. Chem. 22, 931 (2001).

${ }^{33}$ C. Fonseca Guerra, J. G. Snijders, G. te Velde, and E. J. Baerends, Theor. Chem. Acc. 99, 391 (1998).

${ }^{34}$ E. Jan Baerends et al., ADF2017, SCM, Theoretical Chemistry (Vrije Universiteit, Amsterdam, The Netherlands, 2017), https://www.scm.com.

${ }^{35}$ E. van Lenthe, E. J. Baerends, and J. G. Snijders, J. Chem. Phys. 99, 4597 (1993).

${ }^{36}$ E. van Lenthe, E. J. Baerends, and J. G. Snijders, J. Chem. Phys. 101, 9783 (1994).

${ }^{37}$ E. van Lenthe, A. Ehlers, and E.-J. Baerends, J. Chem. Phys. 110, 8943 (1999).

${ }^{38}$ E. van Lenthe and E. J. Baerends, J. Comput. Chem. 24, 1142 (2003).

${ }^{39}$ D. P. Chong, E. van Lenthe, S. van Gisbergen, and E. J. Baerends, J. Comput. Chem. 25, 1030 (2004).

${ }^{40}$ D. P. Chong, Mol. Phys. 103, 749 (2005).

${ }^{41}$ M. Pápai, T. J. Penfold, and K. B. Moller, J. Phys. Chem. C 120, 17234 (2016).

${ }^{42}$ G. Capano, T. J. Penfold, U. Röthlisberger, and I. Tavernelli, Chimia 68, 227 (2014).

${ }^{43}$ J. Eng, C. Gourlaouen, E. Gindensperger, and C. Daniel, Acc. Chem. Res. 48, 809 (2015).

${ }^{44}$ J. E. Subotnik, S. Yeganeh, R. J. Cave, and M. A. Ratner, J. Chem. Phys. 129(24), 244101 (2008).

${ }^{45}$ M. H. Beck, A. Jäckle, G. A. Worth, and H.-D. Meyer, Phys. Rep. 324(1), 1-105 (2000). 